\title{
STABILITY THEOREMS FOR HOLOMORPHIC FOLIATIONS ${ }^{1}$
}

\author{
BY
}

\author{
T. DUCHAMP AND M. KALKA
}

\begin{abstract}
Here we investigate topological stability in the space of holomorphic foliations on a compact manifold. We show that under certain conditions nearby holomorphic foliations are topologically equivalent. We then present examples of foliations which are stable as holomorphic foliations but unstable as smooth foliations.
\end{abstract}

0. Introduction. In [6] Hamilton proved that a $C^{\infty}$-Hausdorff foliation on a compact manifold is differentiably stable provided that the first de Rham cohomology group of a generic leaf is trivial. Epstein and Rosenberg [5] have generalized this result to $C^{r}$-foliations with $r \geqslant 1$ on noncompact manifolds. It is also known [10] that if the first cohomology group of a generic leaf is not trivial then the foliation is not differentiably stable.

It is natural to consider the stability properties of holomorphic foliations (these are foliations on a $C^{\infty}$-manifold which are represented as Haefliger cocycles with coefficients in the pseudogroup of local biholomorphisms of $\mathbf{C}^{q}$ ). The main result of this paper is Theorem 1 which gives conditions under which every holomorphic foliation near a fixed holomorphic foliation is of the same topological type. We do not require that the fixed foliation be Hausdorff; it need only be Riemannian. However, we do require the existence of a closed, locally decomposable form whose restriction to each leaf of the foliation is a volume form. The remainder of this introduction is devoted to the statement of Theorem 1 and to an example.

Let $M$ be an $n$-dimensional, compact, smooth manifold and let $\mathscr{F}$ be a $C^{\infty}$-foliation. Denote by $L \subseteq T M$ the tangential distribution of $\mathscr{F}$, and by $Q$ the normal bundle of $\mathscr{F}$. Finally let $\mathbf{Q}_{\mathscr{F}}$ denote the sheaf of germs of local sections of $Q$ which are locally constant along the leaves of $\mathscr{F}$. We make the following definitions.

DEFINITION. The foliation $\mathscr{F}$ is said to be infinitesimally (differentiably) stable if the sheaf cohomology group $H^{1}\left(M, Q_{\mathscr{F}}\right)$ vanishes. $\mathscr{F}$ is said to be (differentiably) stable if every foliation whose tangential distribution is sufficiently near $L$, in the $C^{\infty}$-topology on sections of the Grassmann bundle, is $C^{\infty}$-conjugate to $\mathscr{F}$. Recall that a foliation $\mathcal{G}$ is $C^{\infty}$-conjugate to $\mathscr{F}$ if there is a diffeomorphism of $M$ sending the leaves of $\mathcal{G}$ to the leaves of $\mathscr{F}$.

Suppose now that $\mathscr{F}$ is a holomorphic foliation. Then there is a complex structure $J$ on the normal bundle $Q$ and there is a notion of holomorphic section which we define in $\S 1$. Let $\theta_{\mathscr{F}}$ denote the sheaf of germs of holomorphic sections of

Received by the editors December 18, 1978.

AMS (MOS) subject classifications (1970). Primary 57D30, 32G99, 58H05.

Key words and phrases. Holomorphic foliations, differentiable stability, deformations.

${ }^{1}$ Research partially supported by NSF grants MCS 78-01826 and MCS78-00399. 
$Q$ which are locally constant along the leaves of $\mathscr{F}$. In analogy with the $C^{\infty}$ case we make the following definitions.

DEFINITION. A holomorphic foliation $\mathscr{F}$ is said to be infinitesimally holomorphically stable if the cohomology group $H^{1}\left(M, \theta_{\mathscr{F}}\right)$ vanishes. $\mathcal{F}$ is said to be holomorphically stable if every holomorphic foliation sufficiently close to $\mathscr{F}$ (in a sense to be made precise in $\$ 2$ ) is holomorphically conjugate to $\mathscr{F}$. A holomorphic foliation $\mathcal{G}$ is holomorphically conjugate to $\mathscr{F}$ if there is a $C^{\infty}$-diffeomorphism of $M$ sending the leaves of $\mathcal{G}$ to the leaves of $\mathscr{F}$ and respecting the complex structures.

In [1] we showed that $H^{1}\left(M, \theta_{\mathcal{F}}\right)$ is finite dimensional and in the case where there is a $C^{\infty}$-foliation transverse to $\mathscr{F}$ we showed that there is a local analytic subspace of $H^{1}\left(M, \theta_{\mathscr{G}}\right)$ which parametrizes conjugacy classes of holomorphic foliations near $\mathscr{F}$. Hence in this case infinitesimal holomorphic stability implies holomorphic stability.

Now it can happen that a holomorphic foliation $\mathscr{F}$ is neither holomorphically stable nor differentiably stable but that every holomorphic foliation sufficiently near $\mathscr{F}$ is $C^{\infty}$-conjugate to $\mathscr{F}$. We will study this phenomenon using the inclusion $i$ : $\boldsymbol{\theta}_{\mathscr{F}} \hookrightarrow \mathbf{Q}_{\mathscr{F}}$ obtained by forgetting the complex structure on $Q$. This map induces a map between cohomology groups

$$
i_{*}: H^{1}\left(M, \theta_{\mathscr{F}}\right) \rightarrow H^{1}\left(M, \mathbf{Q}_{\mathscr{F}}\right) .
$$

DEFINITION. The holomorphic foliation $\mathscr{F}$ is said to be infinitesimally stable under holomorphic deformations provided that the map $i_{*}$ is the zero map. If every holomorphic foliation $\mathcal{G}$ near $\mathscr{F}$ is conjugate to $\mathscr{F}$ via a diffeomorphism of $\boldsymbol{M}$ sending leaves of $\mathcal{G}$ to leaves of $\mathscr{F}$, but not necessarily respecting complex structures, then $\mathscr{F}$ is said to be stable under holomorphic deformations.

The main result of this paper is that infinitesimal stability under holomorphic deformations implies stability under holomorphic deformations. However, our method of proof requires that two restrictions be imposed on $\mathscr{F}$. First we assume that $\mathscr{F}$ is Hermitian. By this we mean that in addition to being a holomorphic foliation $\mathscr{F}$ is also a Riemannian foliation. We must also assume that there is an $\operatorname{SL}(p)$-foliation transverse to $\mathcal{F}$. Here $p=n-2 q$, the dimension of the leaves of $\mathscr{F}$. Recall that an $\operatorname{SL}(p)$-foliation is a foliation whose tangential distribution is given as the kernel of a closed, locally decomposable $p$-form on $M$. Our main result is the following.

TheOREM 1. Let $\mathcal{F}$ be a real codimension-2q Hermitian foliation on a compact manifold $M^{n}$, and suppose there is an $\mathrm{SL}(p)$-foliation transverse to $\mathcal{F}$. If the map

$$
i_{*}: H^{1}\left(M, \theta_{\mathscr{F}}\right) \rightarrow H^{1}\left(M, \mathbf{Q}_{\mathscr{F}}\right)
$$

is the zero map, then $\mathcal{F}$ is stable under holomorphic deformations.

We do not know that the hypotheses of this theorem are all necessary. However, the techniques we use make essential use of both the Hermitian metric and the closed form defining the transverse foliation.

To illustrate the theorem consider the following class of examples. Let $N$ be a 
compact complex manifold and let $M=S^{1} \times N$. Consider the holomorphic foliation $\mathcal{F}$ of $M$ by the circles $\left\{\left(\theta, x_{0}\right): \theta \in S^{1}\right\}, x_{0} \in N$. Let $\pi: M \rightarrow N$ be the projection. Then $\theta_{\mathscr{F}}=\pi^{*} \theta_{N}$ where $\theta_{N}$ is the sheaf of germs of holomorphic vector fields on $N$. Further, $\mathbf{Q}_{\mathscr{F}}$ is the pull back under $\pi$ of the sheaf of germs of vector fields on $N$. An application of the results in $\$ 3$ shows that there is a commutative diagram

$$
\begin{aligned}
& H^{1}\left(M, \theta_{\mathscr{F}}\right) \\
& \text { त } \uparrow \\
& \stackrel{i *}{\rightarrow} \quad H^{1}\left(M, Q_{\mathscr{F}}\right) \\
& \text { ॥ } \uparrow \\
& H^{1}\left(N, \theta_{N}\right) \oplus H^{1}\left(S^{1}, \mathbf{C}\right) \otimes_{\mathrm{C}} \Gamma\left(N, \theta_{N}\right) \quad \stackrel{j_{*}}{\rightarrow} \quad H^{1}\left(S^{1}, \mathbf{R}\right) \otimes_{\mathbf{R}} \Gamma(N, T N)
\end{aligned}
$$

where $j_{*}$ is the map obtained by composing the projection onto $H^{1}\left(S^{1}, \mathbf{C}\right) \otimes_{\mathrm{C}} \Gamma\left(N, \theta_{N}\right) \cong H^{1}\left(S^{1}, \mathbf{R}\right) \otimes_{\mathrm{R}} \Gamma\left(N, \theta_{N}\right)$ with the map induced by the inclusion $\theta_{N} \hookrightarrow$ TN. Hence $\mathscr{F}$ is infinitesimally stable under holomorphic deformations if and only if $\Gamma\left(N, \theta_{N}\right)=0$. Observe that if $X \in \Gamma\left(N, \theta_{N}\right)$ we can use the 1-parameter family of biholomorphisms of $N$ generated by $X$ to obtain, via suspension, a family of holomorphic foliations on $M$ which is differentiably nontrivial. Theorem 1 shows that the only way that such a family can arise is through elements of $\Gamma\left(N, \theta_{N}\right)$, hence if $N$ is a hyperbolic manifold then $\mathcal{F}$ is stable under holomorphic deformations. Since $H^{1}\left(S^{1}, \mathbf{R}\right) \neq 0$ the foliation $\mathscr{F}$ is certainly not stable.

The paper is organized as follows. In $\$ 1$ we construct resolutions of the sheaves $\boldsymbol{\theta}_{\mathscr{F}}$ and $\mathbf{Q}_{\mathscr{F}}$ that are needed in the proof of Theorem 1. In $\$ 2$ we prove Theorem 1 . In $\$ 3$ we give a class of holomorphic foliations where the map $i_{*}: H^{1}\left(M, \theta_{\S}\right) \rightarrow$ $H^{1}\left(M, \mathbf{Q}_{\mathscr{g}}\right)$ is computable.

Notation. We will use the Einstein summation convention throughout. Also if $E \rightarrow M$ is a vector bundle we will denote by $\mathbf{E}$ the sheaf of germs of sections of $E$ and if $\mathcal{S}$ is a sheaf on $M$ we will denote the space of global sections of $\mathcal{S}$ by $\Gamma(\mathcal{S})$. If $V$ is any bundle associated to the foliation $\mathscr{F}$ then $\mathbf{V}_{\mathscr{F}}$ will be used to denote the sheaf of sections of $V$ which are locally constant along the leaves of $\mathscr{F}$.

1. The resolution of the sheaf $\theta_{\mathscr{G}}$. Throughout this paper $M$ is assumed to be a compact, oriented $C^{\infty}$-manifold of dimension $n$ and $\mathscr{F}$ is a real codimension- $2 q$ Hermitian foliation on $M . \mathscr{F}^{\perp}$ will denote an $\operatorname{SL}(p)$ foliation transverse to $\mathscr{F}$, where $p=n-2 q$. To every such structure there is associated a distinguished family of charts defined as follows.

Definition. An adapted coordinate chart for $M$ is a chart $\psi: U \subseteq M \rightarrow \mathbf{R}^{p} \times \mathbf{C}^{q}$ with coordinate functions $(x, z)=\left(x^{1}, \ldots, x^{p}, z^{1}, \ldots, z^{q}\right)$ such that the restriction of $\mathscr{F}$ to $U$ is given by the fibres of the map $(x, z) \mapsto z$, and $\mathscr{F}^{\perp}$ restricted to $U$ is given by the fibres of the map $(x, z) \mapsto x$. We will use adapted coordinates exclusively, using Latin indices to range from 1 to $p$, and Greek indices will range from 1 to $q$.

On overlapping charts $(x, z) \in U$ and $(\tilde{x}, \tilde{z}) \in \tilde{U}$ the adapted coordinates are related as follows: $\tilde{x}=f(x), \tilde{z}=g(z)$ with $f$ of class $C^{\infty}$ and $g$ holomorphic. 
Using adapted coordinates we see that the complexified cotangent bundle of $M$ splits as follows.

$$
T M_{\mathbf{C}}^{*}=L_{\mathbf{C}}^{*} \oplus Q^{(0,1)^{*}} \oplus Q^{(1,0)^{*}}
$$

where $L_{\mathrm{C}}^{*}$ is generated by the forms $d x^{i}, Q^{(1,0)^{*}}$ by the forms $d z^{\alpha}$ and $Q^{(0,1)^{*}}$ by the forms $d \bar{z}^{\alpha}$.

Two subcomplexes of the complexified de Rham complex of $M$ are relevant to the study of deformation theory for holomorphic foliations. Denote by $\left(L_{Q}^{*}, d_{\|}\right)$the sheaf complex defined as follows. Let $L_{Q}^{* s}$ denote the sheaf of germs of sections of the bundle $\Lambda^{s}\left(L_{\mathbf{C}}^{*}\right) \otimes Q^{1,0}$ and let $d_{\|}: \mathbf{L}_{Q}^{* s} \rightarrow L_{Q}^{* s+1}$ be the operator defined by the local formula

$$
d_{\|}\left(\omega_{I}^{\alpha} d x^{I} \otimes \frac{\partial}{\partial z^{\alpha}}\right)=\frac{\partial \omega_{I}^{\alpha}}{\partial x^{i}} d x^{i} \wedge d x^{I} \otimes \frac{\partial}{\partial z^{\alpha}}
$$

where $I=\left(i_{1}, \ldots, i_{s}\right)$ is a multi-index and $d x^{I}=d x^{i_{1}} \wedge \cdots \wedge d x^{i_{s}}$.

The sheaf

$$
\mathbf{Q}_{\mathscr{F}}^{1,0}=\operatorname{Ker}\left\{\mathbf{L}_{\mathcal{Q}}^{* 0} \stackrel{d_{\|}}{\rightarrow} \mathbf{L}_{\mathcal{Q}}^{* 1}\right\}
$$

is the sheaf of germs of sections of $Q^{(1,0)}$ of the form $\xi=\xi^{\alpha}(z)\left(\partial / \partial z^{\alpha}\right)$ where $\xi^{\alpha}(z)$ are smooth functions of $z$ only.

As a real vector bundle $Q^{(1,0)}$ is isomorphic to the real vector bundle $Q=$ $T M / L$. In local coordinates this isomorphism is given by the assignments $\partial / \partial z^{\alpha} \mapsto$ $\partial / \partial u^{\alpha}$ and $i\left(\partial / \partial z^{\alpha}\right) \mapsto-\partial / \partial v^{\alpha}$ where $z^{\alpha}=u^{\alpha}+i v^{\alpha}$. Under this identification $\mathbf{Q}_{\mathcal{F}}^{(1,0)}$ is the sheaf $\mathbf{Q}_{\mathscr{F}}$ of germs of sections of $Q$ which are locally constant along the leaves of $\mathscr{F}$ and $\left(\mathbf{L}^{*}, d_{\|}\right)$is a resolution of $\mathbf{Q}_{\mathscr{F}}$ This resolution was constructed independently in [6], [7] and [8].

Denote by $\mathbf{A}_{Q}^{* s}$ the sheaf of germs of sections of the vector bundle

$$
\Lambda^{s}\left(L_{\mathbf{C}} \oplus Q^{(0,1)}\right) * \otimes Q^{(1,0)}
$$

and let $d_{A}: \mathbf{A}_{Q}^{* s} \rightarrow \mathbf{A}_{Q}^{* s+1}$ be the operator $d_{A}=d_{\|}+\bar{\partial}$ where $d_{\|}$and $\bar{\partial}$ are given by the local formulae

$$
d_{\|}\left(\omega_{I A}^{\alpha} d x^{I} \wedge d \bar{z}^{A} \otimes \frac{\partial}{\partial z^{\alpha}}\right)=\frac{\partial \omega_{I A}^{\alpha}}{\partial x^{i}} d x^{i} \wedge d x^{I} \wedge d \bar{z}^{A} \otimes \frac{\partial}{\partial z^{\alpha}}
$$

and

$$
\bar{\partial}\left(\omega_{I A}^{\alpha} d x^{I} \wedge d \bar{z}^{A} \otimes \frac{\partial}{\partial z^{\alpha}}\right)=(-1)^{|I|} \frac{\partial \omega_{I A}^{\alpha}}{\partial \bar{z}^{\beta}} d x^{I} \wedge d \bar{z}^{\beta} \wedge d \bar{z}^{A} \otimes \frac{\partial}{\partial z^{\alpha}}
$$

where $I$ and $A$ are multi-indices with $|I|+|A|=s$. Note that the sheaf $\theta_{\mathscr{F}}$ defined by

$$
\boldsymbol{\theta}_{\mathscr{F}}=\operatorname{Ker}\left\{d_{A}: \mathbf{A}_{Q}^{* 0} \rightarrow \mathbf{A}_{Q}^{* 1}\right\}
$$

is the sheaf of germs of sections of $Q^{(1,0)}$ having the local form $\xi=\xi^{\alpha} \partial / \partial z^{\alpha}$ where the $\xi^{\alpha}$ are holomorphic functions of $\left(z^{1}, \ldots, z^{p}\right)$. In [1] we showed, for a more general class of holomorphic foliations, that $\left(\mathbf{A}_{\boldsymbol{Q}}^{*}, d_{A}\right)$ is a resolution of $\boldsymbol{\theta}_{\mathscr{F}}$ 
Observe that the inclusion $L_{\mathbf{C}} \hookrightarrow L_{\mathbf{C}} \otimes Q^{(0,1)}$ induces a map of complexes of sheaves

$$
i:\left(\mathrm{A}_{Q}^{*}, d_{A}\right) \rightarrow\left(\mathrm{L}_{Q}^{*}, d_{\|}\right)
$$

This map gives rise to the map

$$
i_{*}: H \cdot\left(M, \theta_{\mathscr{F}}\right) \rightarrow H \cdot\left(M, \mathbf{Q}_{\mathscr{F}}\right)
$$

which relates holomorphic invariants of $\mathscr{F}$ to differential invariants of $\mathscr{F}$. This map is the map used in the introduction to define infinitesimal stability under holomorphic deformations.

2. Infinitesimal stability implies stability. In this section we will prove Theorem 1 using techniques inspired by Reinhart [11], [12] together with the deformation theory presented in [1].

Definition. A form $\phi \in \mathbf{A}_{Q}^{* r}$ is said to be baselike if in adapted coordinates it is of the form $\phi=\phi_{B}^{\alpha}(z) d \bar{z}^{B} \otimes \partial / \partial z^{\alpha}$ where $\phi_{B}^{\alpha}$ depends only on $z$. We denote the sheaf of baselike $s$-forms by $\mathbf{A}_{b}^{* s}$. From the local formula for the operator $d_{A}$ it is clear that it restricts to an operator on the sheaf of baselike forms, which we will denote by $d_{b}$. Note that if $\phi$ is baselike then $d_{\|} \phi=0$ so $d_{b} \phi=\bar{\partial} \phi$.

We can now give the following characterization of infinitesimal stability.

Proposition 1. Infinitesimal (differentiable) stability under holomorphic deformations is equivalent to the condition that every element in $H^{1}\left(M, \theta_{\mathcal{G}}\right)$ has a baselike representative.

Proof. Suppose $\tau=\eta+\tilde{\eta}$ where $\eta=\eta_{i}^{\alpha} d x^{i} \otimes \partial / \partial z^{\alpha}$ and $\tilde{\eta}=\eta_{\beta}^{\alpha} d z^{\beta} \otimes \partial / \partial z^{\alpha}$ is a representative for a class in $H^{1}\left(M, \theta_{G}\right)$. Then since $d_{\|} \eta=0$, infinitesimal stability implies that $\eta=d_{\|} \xi$ for some vector field $\xi \in \Gamma\left(M, Q^{(1,0)}\right)$. Hence $\tau$ is cohomologous to $\omega=\tau-d_{A} \xi=\eta-\bar{\partial} \xi \in \Gamma\left(M, Q^{(0,1)^{*}} \otimes Q^{(1,0)}\right)$. But $d_{A} \tau=0$ so $d_{\|} \omega=\bar{\partial} \omega=0$, which shows that $\omega$ is baselike. The converse follows by reversing the argument.

Since the foliation $\mathscr{F}$ is Hermitian and $\mathscr{F}^{\perp}$ is an $\operatorname{SL}(p)$ foliation there is a metric on $M$ of the form

$$
d s^{2}=g_{i j}(x, z) d x^{i} \otimes d x^{j}+h_{\alpha \bar{\beta}}(z) d z^{\alpha} \otimes d \bar{z}^{\beta}
$$

with $g=\sqrt{\operatorname{det} g_{i j}}$ a function of $x$ only and $h=\sqrt{\operatorname{det} h_{\alpha \bar{\beta}}}$ and $h_{\alpha \beta}$ functions of $z$ only.

We wish to use this metric to define an inner product, $\langle$,$\rangle , on the space of$ global sections of $\mathbf{A}_{Q}^{* *}$. Let ${ }^{*}$ denote the usual Hodge star operator on the complexified de Rham complex $\Omega_{\mathrm{C}}(M)$ of $M$ with respect to the metric $d s^{2}$. This operator extends to an operator

$$
* \Gamma\left(\mathbf{A}_{Q}^{* \cdot}\right) \rightarrow \Gamma\left(\mathbf{A}_{Q}^{* n-\cdot}\right)
$$

defined by ${ }^{*}(\omega \otimes \xi)=\left({ }^{*} \omega\right) \otimes \xi$.

Define a bilinear product

$$
\bar{\wedge}: \Gamma\left(\mathbf{A}_{Q}^{* r}\right) \otimes \Gamma\left(\mathbf{A}_{Q}^{* s}\right) \rightarrow \Omega_{\mathbf{C}}^{r+s}(M)
$$


by

$$
\tilde{\omega} \bar{\wedge} \tilde{\phi}=\langle\xi, \eta\rangle \omega \wedge \boldsymbol{\phi}
$$

for $\tilde{\omega}=\omega \otimes \xi, \tilde{\phi}=\phi \otimes \eta$ global sections of $\mathbf{A}_{Q}^{*}$ where $\langle\xi, \eta\rangle$ is the inner product of the vector fields $\xi$ and $\eta$. Then the inner product on $\Gamma\left(A_{Q}^{* *}\right)$ is given by

$$
\langle\tilde{\omega}, \tilde{\phi}\rangle= \begin{cases}0 & \text { for } r \neq s, \\ \int \tilde{\omega} \bar{\wedge} \tilde{\varphi} & \text { for } r=s,\end{cases}
$$

where $\tilde{\boldsymbol{\omega}} \in \Gamma\left(\mathbf{A}_{Q}^{* r}\right), \tilde{\phi} \in \Gamma\left(\mathbf{A}_{Q}^{* s}\right)$. Denote by $A_{Q}^{*}$ the Hilbert space completion of $\Gamma\left(A_{Q}^{*}\right)$ and by $A_{b}^{*}$ the closure of the subspace of baselike forms.

The complex $\left(A_{Q}^{* *}, d_{A}\right)$ being elliptic [1], there is a Hodge theory associated to it. Specifically, denote the adjoint of $d_{A}$ by $d_{A}^{*}$, and let $\Delta_{A}=d_{A} d_{A}^{*}+d_{A}^{*} d_{A}$. Then $\left(\operatorname{Ker} \Delta_{A}\right)^{\cdot} \subseteq \Gamma\left(A_{Q}^{*}\right)$ and the map $\left(\operatorname{Ker} \Delta_{A}\right)^{*} \rightarrow H \cdot\left(M, \theta_{\mathcal{G}}\right)$ taking a harmonic form to its representative is an isomorphism. Here $\left(\operatorname{Ker} \Delta_{A}\right)^{s}$ denotes the kernel of $\Delta_{A}$ as an operator on $A_{Q}^{* s}$.

Let the adjoint of $d_{b}$ be denoted by $d_{b}^{*}$ and set $\Delta_{b}=d_{b} d_{b}^{*}+d_{b}^{*} d_{b}$. We need the following lemma.

LEMMA 1. (1) $\left.d_{A}^{*}\right|_{A_{b}^{*}}=d_{b}^{*}$ and

(2) $\left.\Delta_{A}\right|_{A_{b}^{*}}=\Delta_{b}$.

In particular, $\Delta\left(A_{b}^{* *}\right) \subset A_{b}^{* *}$. Here $\left.\right|_{A_{b}^{*}}$ denotes the restriction to the subspace $A_{b}^{* *} \subseteq$ $A_{Q}^{*}$.

To prove this result it is necessary to find a formula for $d_{A}^{*}$. First let $E$ : $\Gamma\left(\mathbf{A}_{Q}^{* 0}\right) \rightarrow \Gamma\left(\mathbf{A}_{Q}^{* 1}\right)$ be the operator defined by

$$
E\left(\eta^{\alpha} \otimes \frac{\partial}{\partial z^{\alpha}}\right)=h^{\beta \bar{\alpha}} \frac{\partial}{\partial z^{\alpha}}\left(h_{\beta \bar{\delta}} \eta^{\delta}\right) d \bar{z}^{\gamma} \otimes \frac{\partial}{\partial z^{\alpha}}
$$

where $\left(h^{\bar{\beta} \alpha}\right)$ is the inverse matrix of $\left(h_{\bar{\beta} \alpha}\right)$.

LEMMA 2. If $\tilde{\phi}=\phi \otimes \eta$ then

$$
d_{A}^{*} \tilde{\phi}= \pm(* d * \phi) \otimes \eta-*(* \phi \wedge E(\eta)) \text {. }
$$

Proof. Let $\tilde{\omega}=\omega \otimes \xi, \tilde{\phi}=\phi \otimes \eta$ be in $\Gamma\left(\mathbf{A}_{Q}^{*(r-1)}\right)$ and $\Gamma\left(\mathbf{A}_{Q}^{* r}\right)$ respectively. Then since $* \phi$ contains the factor $d z^{1} \wedge \cdots \wedge d z^{q}$, it follows that $d_{A}(\langle\xi, \eta\rangle \omega \wedge * \phi)=$ $d(\langle\xi, \eta\rangle \omega \wedge * \phi)$ where $d$ is ordinary exterior differentiation. So by Stokes' Theo$\operatorname{rem} 0=\int_{M} d_{A}(\langle\xi, \eta\rangle \omega \wedge * \phi)$.

Now apply the product rule to rewrite this equation in the form

$$
\begin{aligned}
0= & \int_{M}\left(d_{A}(\omega \otimes \xi)\right) \bar{\wedge}(* \phi \otimes \eta)+\int_{M}(\omega \otimes \xi) \bar{\wedge}(d * \phi) \otimes \eta \\
& +\int_{M}(\omega \otimes \xi) \bar{\wedge}(* \phi \wedge E(\eta)) \\
= & \left\langle d_{A} \tilde{\omega}, \tilde{\phi}\right\rangle \mp\langle\tilde{\omega},(* d * \phi) \otimes \eta+*(* \phi \wedge E(\eta))\rangle,
\end{aligned}
$$

completing the proof of Lemma 2. 
To prove Lemma 1 we observe that a straightforward calculation shows that if $\tilde{\phi}=\phi \otimes \eta$ is baselike, then $(* d * \phi) \otimes \eta$ and $*(* \phi \wedge E(\eta))$ are baselike. The key facts here are that $h_{\alpha \bar{\beta}}$ depends only on $z$, and that the volume form on $M$ can be written

$$
\left(h(z) d z^{1} \wedge \cdots \wedge d z^{q} \wedge d \bar{z}^{1} \wedge \cdots \wedge d \bar{z}^{q}\right) \wedge\left(g(x) d x^{1} \wedge \cdots \wedge d x^{p}\right) .
$$

This shows that $d_{A}^{*}\left(A_{b}^{*}\right) \subseteq A_{b}^{*}$; completing the proof of Lemma 1 .

Since $\Delta_{A}$ is selfadjoint and Fredholm, so is $\Delta_{b}$, its restriction to the closed subspace $A_{b}^{* *} \subset A_{Q}^{* *}$. Let

$$
H_{A}: A_{Q}^{* \cdot} \rightarrow\left(\operatorname{Ker} \Delta_{A}\right)^{*}, \quad H_{b}: A_{b}^{* \cdot} \rightarrow\left(\operatorname{Ker} \Delta_{b}\right)^{\prime}
$$

be the projection operators onto $\left(\operatorname{Ker} \Delta_{A}\right)$ and $\left(\operatorname{Ker} A_{b}\right)^{\cdot}$ and let $G_{A}$ and $G_{b}$ be the associated Green's operators. Then $I=\Delta_{A} G_{A}+H_{A}, I=\Delta_{b} G_{b}+H_{b}$.

The following is a corollary to Lemma 1.

LEMMA 3. (1) $H_{b}=H_{A \mid A_{b}^{*}}$.

(2) $G_{b}=G_{A \mid A_{b}^{*}}$

Proof. (1) Let $\omega \in A_{b}^{* s}$; recall that $H_{A} \omega$ is the unique element of $\left(\operatorname{Ker} \Delta_{A}\right)^{s}$ cohomologous in $A_{Q}^{* \cdot}$ to $\omega$. But $\omega-H_{b} \omega=d_{b} \eta$ for some element $\eta \in A_{b}^{* *} . H_{b} \omega \in$ $\left(\operatorname{Ker} \Delta_{b}\right)^{s} \subseteq\left(\operatorname{Ker} \Delta_{A}\right)^{s}$ and $d_{b} \eta=d_{A} \eta$. Hence $H_{b} \omega=H_{a} \omega$.

(2) Recall that $\Delta_{b}$ is invertible on $\left(\operatorname{Ker} \Delta_{b}\right)^{\perp} \subseteq A_{b}^{* \cdot}$ and that

$$
G_{b} \omega=\Delta_{b}^{-1}\left(\omega-H_{b} \omega\right) \text { for } \omega \in A_{b}^{* *} \text {. }
$$

Similarly, $G_{A} \omega=\Delta_{A}^{-1}\left(\omega-H_{A} \omega\right)$ for $\omega \in A_{Q}^{* *}$. Therefore by Lemma $1(2)$ and Lemma 3(1) we have the chain of equalities

$$
G_{b} \omega=\Delta_{b}^{-1}\left(\omega-H_{b} \omega\right)=\Delta_{A}^{-1}\left(\omega-H_{A} \omega\right)=G_{A} \omega
$$

for $\omega \in A_{b}^{*}$.

LEMMA 4. Let $\mathcal{F}$ be infinitesimally stable under holomorphic deformations. Then $\left(\operatorname{Ker} \Delta_{b}\right)^{1}=\left(\operatorname{Ker} \Delta_{A}\right)^{1}$.

Proof. We know from Lemma 1 that $\left(\operatorname{Ker} \Delta_{b}\right)^{1} \subseteq\left(\operatorname{Ker} \Delta_{A}\right)^{1}$. To prove the reverse inclusion pick an element $\omega \in\left(\operatorname{Ker} \Delta_{A}\right)^{1}$. By Proposition 1 there is a $d_{A}$-closed baselike form $\omega_{1}$ cohomologous in $A_{Q}^{*}$ to $\omega$. Hence $\omega=H_{A}(\omega)=H_{A}\left(\omega_{1}\right)$ and by Lemma 3(1) $H_{A}\left(\omega_{1}\right)=H_{b}\left(\omega_{1}\right)$. Therefore $\omega=H_{b}\left(\omega_{1}\right) \in\left(\operatorname{Ker} \Delta_{b}\right)^{2}$.

We can now begin the proof of Theorem 1. First observe that an element $\phi=\operatorname{Hom}\left(L_{\mathrm{C}} \oplus Q^{(0,1)}, Q^{(1,0)}\right)$ defines a distribution $L_{\phi}$ and a complex structure on its normal bundle $Q$ as follows. Write $\phi=\phi_{L}+\phi_{Q}$ where $\phi_{L} \in \operatorname{Hom}\left(L_{\mathrm{C}}, Q^{(1,0)}\right) \cong$ $\operatorname{Hom}_{\mathrm{R}}(L, Q)$ and $\phi_{Q} \in \operatorname{Hom}_{\mathrm{C}}\left(Q^{(0,1)}, Q^{(1,0)}\right)$. Then using the decomposition $T M=$ $L \oplus Q$ induced by the local product structure of $M$, set $L=\left\{X+\phi_{L}(X) \mid X \in L\right\}$. It is also clear that $\phi_{Q}$ defines a complex structure $J_{\phi}$ on $Q$ in a similar way.

In [1] we showed that the pair $\left(L_{\phi}, J_{\phi}\right)$ defines a holomorphic foliation $\mathscr{F}_{\phi}$ provided that $\phi$ satisfies the differential equation $D \phi=d_{A} \phi-[\phi, \phi]=0$, where $[$,$] is a natural extension of the bracket operation used in the deformation theory$ of complex manifolds, and reduces to that bracket when restricted to baselike forms. Now put the $C^{\infty}$ topology on the space $\Gamma\left(\mathbf{A}_{Q}^{* 1}\right)$. This induces a topology on 
the space of holomorphic foliations. It is this topology to which we alluded in the introduction.

Observe that if $\phi_{L}=0$ then the tangent bundle of the foliation $\mathscr{F}_{\phi}$ is just $L$ and hence $\mathscr{F}_{\phi}$, considered as a $C^{\infty}$-foliation, is identical to $\mathscr{F}$. This fact will be needed in the proof of Theorem 2.

We will also need the following result, also proved in [1]. The set of solutions of small norm of the equations $\phi=\phi_{0}+d_{A}^{*} G_{A}[\phi, \phi]$ and $H_{A}[\phi, \phi]=0$ for $\phi_{0} \in$ $\operatorname{Ker} \Delta_{A}, \phi_{0}$ of small norm, parametrizes a family of holomorphic foliations near $\mathcal{F}$. Furthermore, every holomorphic foliation near $\mathscr{F}$ is conjugate, via a diffeomorphism of $M$ respecting complex structures to a member of this family. Hence, to complete the proof of Theorem 1 it is sufficient to show that every solution $\phi$ of the equation $\phi=\phi_{0}+d_{A}^{*} G_{A}[\phi, \phi]$ with $\phi_{0} \in\left(\operatorname{Ker} \Delta_{A}\right)^{1}$ of small norm is baselike for if $\phi$ is baselike then $\phi_{L}=0$.

But solutions of this equation are constructed by iteration with $\phi=\lim \phi_{n}$ and $\phi_{n+1}=\phi_{0}+d_{A}^{*} G_{A}\left[\phi_{n}, \phi_{n}\right]$. If $\phi_{n}$ is baselike then $\left[\phi_{n}, \phi_{n}\right]$ is baselike and by Lemmas 1 and 3 the form $d_{A}^{*} G_{A}\left[\phi_{n}, \phi_{n}\right]$ is also baselike. Hence $\phi_{n}$ baselike implies $\phi_{n+1}$ baselike. Since by Lemma $4, \phi_{0}$ is baselike it follows by induction that all $\phi_{n}$ are baselike and therefore so is their limit $\phi$.

3. The computation of the map $i_{*}$. In this section we prove a generalization of the Leray-Hirsch theorem and use it to compute the map $i_{*}$. We then show that in certain cases stability under holomorphic deformations is equivalent to the condition $\Gamma\left(M, \theta_{\mathscr{G}}\right)=0$.

We begin with a definition.

Definition. A Hausdorff holomorphic foliation $\mathscr{F}$ on a manifold $M$ is called a cohomology product foliation if there are forms $\omega_{1}, \ldots, \omega_{r}$ on $M$ with $d \omega_{i} \in$ $\Gamma\left(Q^{(1,0)^{*}} \wedge \Lambda \cdot T^{*} M_{\mathrm{C}}\right)$ for $i=1, \ldots, r$ and such that for each leaf $N \stackrel{j}{\hookrightarrow} M$ of $\mathscr{F}$ and every integer $1 \leqslant s \leqslant \operatorname{dim} N$ there is an isomorphism $H^{s}(N, \mathbf{R}) \simeq$ $\Lambda^{s}\left(j^{*} \omega_{1}, \ldots, j^{*} \omega_{r}\right)_{n}$ where $\Lambda\left(j^{*} \omega_{1}, \ldots, j^{*} \omega_{r}\right)_{n}$ denotes the free graded commutative algebra generated by $j^{*} \omega_{1}, \ldots, j^{*} \omega_{r}$ truncated at degree $n=\operatorname{dim} N$. The set $\left\{\omega_{1}, \ldots, \omega_{r}\right\}$ is called a global cohomology basis for the leaves of $\mathscr{F}$.

This definition is motivated by the following version of the Leray-Hirsch theorem.

THEOREM. Let $\mathcal{F}$ be as in the previous definition. Then there is a commutative diagram

$$
\begin{array}{ccc}
H \cdot\left(M, \theta_{\mathscr{F}}\right) & \stackrel{i^{*}}{\rightarrow} & H \cdot\left(M, \mathbf{Q}_{\mathcal{F}}\right) \\
\phi_{*} \uparrow & & \psi_{*} \uparrow \\
H \cdot\left(\Gamma\left(A_{b}^{* *}\right), d_{b}\right) \otimes \Lambda \cdot\left(\omega_{1}, \ldots, \omega_{r}\right)_{n} & \stackrel{j^{*}}{\rightarrow} & \Gamma\left(\mathbf{Q}_{\mathcal{F}}\right) \otimes \Lambda \cdot\left(\omega_{1}, \ldots, \omega_{r}\right)_{n}
\end{array}
$$

where the vertical arrows are isomorphisms and where $j_{*}$ is the zero map on $H^{s}\left(\Gamma\left(A_{b}^{*}\right), d_{b}\right) \otimes \Lambda \cdot\left(\omega_{1}, \ldots, \omega_{r}\right)_{n}$ for $s>1$ and for $s=0$ is given by the inclusion

$$
\Gamma\left(\theta_{\mathscr{F}}\right) \otimes \Lambda \cdot\left(\omega_{1}, \ldots, \omega_{r}\right)_{n} \stackrel{i \otimes i d}{\hookrightarrow} \Gamma\left(\mathbf{Q}_{\mathscr{F}}\right) \otimes \Lambda \cdot\left(\omega_{1}, \ldots, \omega_{r}\right)_{n}
$$


Proof. Consider the following commutative diagram of complexes which in cohomology induces the above diagram.

$$
\begin{array}{ccc}
\left(\Gamma\left(A_{\mathcal{Q}}^{* \cdot}\right), d_{A}\right) & \stackrel{i}{\rightarrow} & \left(\Gamma\left(L_{Q}^{*}\right), d_{\|}\right) \\
\psi & & \\
\left(\Gamma\left(A_{b}^{* *}\right), d_{b}\right) \otimes\left(\Lambda \cdot\left(\omega_{1}, \ldots, \omega_{r}\right)_{n}\right. & \stackrel{j}{\rightarrow} & \Gamma\left(\mathbf{Q}_{\mathcal{F}}\right) \otimes\left(\Lambda \cdot\left(\omega_{1}, \ldots, \omega_{r}\right)_{n}, d_{0}\right)
\end{array}
$$

where $d_{0}$ is the zero differential and $j$ is defined in the obvious manner. It suffices to show that $\phi$ and $\psi$ induce isomorphisms in cohomology. We will prove this for $\phi$ (the result for $\psi$ is proved in a similar way).

Observe that $\Gamma\left(A_{Q}^{* *}\right)$ is equipped with the bigrading

$$
\Gamma\left(A_{Q}^{* \cdot}\right)^{s, t}=\Gamma\left(Q^{(0, s)^{*}} \otimes \Lambda^{t} L^{*} \otimes Q^{(1,0)}\right)
$$

and is a filtered complex with respect to the decreasing filtration

$$
F^{u} \Gamma\left(A_{Q}^{* \cdot}\right)=\bigoplus_{s>u} \Gamma\left(A_{Q}^{*}\right)^{s, \cdot}
$$

There is also a filtration

$$
F^{u} \Gamma\left(A_{b}^{* *}\right) \otimes \Lambda \cdot\left(\omega_{1}, \ldots, \omega_{r}\right)_{n}=\bigoplus_{s>u} \Gamma\left(A_{b}^{* s}\right) \otimes \Lambda \cdot\left(\omega_{1}, \ldots, \omega_{r}\right)_{n}
$$

and $\phi$ is filtration preserving. By the comparison theorem it is sufficient to show that the following map at the $E_{1}$ level of the associated spectral sequences is an isomorphism

$$
E_{1}(\phi)^{s, t}: \Gamma\left(A_{b}^{* s}\right) \otimes \Lambda^{t}\left(\omega_{1}, \ldots, \omega_{r}\right)_{n} \rightarrow H^{t}\left(M, Q_{\xi}^{(0, s)^{*}} \otimes Q_{\xi}^{(1,0)}\right)
$$

where $H^{t}\left(M, \mathrm{Q}_{\mathscr{F}}^{(0, s)^{*}} \otimes \mathrm{Q}_{\mathscr{F}}^{(1,0)}\right)=H^{t}\left(\Gamma\left(A^{* \cdot}\right)^{s,}, d_{\|}\right)$which follows from the fact that $\left(A_{Q}^{* s, \cdot}, d_{\|}\right)$is a resolution of $Q_{\mathscr{G}}^{(0, s)^{*}} \otimes Q_{\mathcal{F}}^{(1,0)}$. See [1, Proposition 4.7].

To show that $E_{1}(\phi)$ is an isomorphism we need the following property of Hausdorff foliations. Let $N_{0}$ denote the generic leaf of $\mathscr{F}$ and let $D^{q}$ be the unit disc in $C^{q}$. Denote by $U_{0}$ the space $N_{0} \times D^{q}$ and by $\mathscr{F}_{0}$ the holomorphic foliation given by the sets $N_{0} \times p$ for $p \in D^{q}$. Let $N$ be any leaf of $\mathscr{F}$ then there is a tubular neighborhood $U$ of $N$ which is a union of leaves of $\mathcal{F}$ and a finite cover $\pi_{U}$ : $U_{0} \rightarrow U$ respecting holomorphic foliations. Since $\mathscr{F}_{0}$ is a product foliation and $\mathbf{Q}_{\mathcal{F}_{0}}^{(0, s)^{*}} \otimes \mathbf{Q}_{\mathcal{F}_{0}}^{(1,0)}$ is a pullback under the projection $U_{0} \mapsto D^{q}$ of a sheaf on $D^{q}$ it follows from the Künneth formula that in this special case there is an isomorphism,

$$
E_{1}(\phi): \Gamma\left(U_{0}, \mathbf{Q}_{\mathcal{F}}^{(0, s)^{*}} \otimes \mathbf{Q}_{\xi}^{(1,0)}\right) \otimes \Lambda^{t}\left(\omega_{1}, \ldots, \omega_{r}\right)_{n} \rightarrow H^{t}\left(\Gamma\left(V_{0,} A^{*, \cdot ;}\right), d_{\|}\right)
$$

and by averaging over the finite group of deck transformations of $\pi_{U}$ this isomorphism descends to an isomorphism

$$
E_{1}(\phi)_{\mid U}^{s, t}: \Gamma\left(U, \mathbf{Q}_{\mathcal{F}}^{(0, s)^{*}} \otimes \mathbf{Q}_{\mathcal{F}}^{(1,0)}\right) \otimes \Lambda^{t}\left(\omega_{1}, \ldots, \omega_{r}\right)_{n} \stackrel{\sim}{\rightarrow} H^{t}\left(\Gamma\left(U, A^{* s,}\right), d_{\|}\right) .
$$

This implies that $E_{1}(\phi)$ is injective. For suppose that $\phi(\omega)=d_{\|} \tau$ for some nonzero element $\omega \in \Gamma\left(A_{b}^{* s}\right) \otimes \Lambda^{t}\left(\omega_{1}, \ldots, \omega_{r}\right)_{n}$. Then $\omega \neq 0$ on some set $U$ as above. But then $\omega_{\mid U}=d_{\|} \tau_{\mid U}$ contradicting the fact that $E_{1}(\phi)_{\mid U}$ is an isomorphism. 
To see that $E_{1}(\phi)$ is surjective let $\left\{U_{\alpha}\right\}$ be a finite cover of $M$ by tubular neighborhoods as above and let $\left\{f_{\alpha}\right\}$ be a partition of unity subordinate to $\left\{U_{\alpha}\right\}$ consisting of $C^{\infty}$-functions which are constant along the leaves of $\mathscr{F}$. Let $\omega \in$ $\Gamma\left(A_{Q}^{* *}\right)^{s, t}$ be a $d_{\|}$-closed form. Then by the above results $\omega_{\mid U_{\alpha}}=\omega_{\alpha}+d_{\|} \tau_{\alpha}$ where $\omega_{\alpha} \in \Gamma\left(U_{\alpha}, A_{b}^{* s}\right) \otimes \Lambda^{t}\left(\omega_{1}, \ldots, \omega_{r}\right)_{n}$. Note that since $f_{\alpha}$ is constant along the leaves of $\mathscr{F}, d_{\|} f_{\alpha}=0$. Hence, $d_{\|} \tau=d_{\|}\left(\Sigma_{\alpha} f_{\alpha} \tau_{\alpha}\right)=\Sigma_{\alpha} f_{\alpha} d_{\|} \tau_{\alpha}$. Therefore,

$$
\omega=\sum_{\alpha} f_{\alpha} \omega=\sum_{\alpha} f_{\alpha}\left(\omega_{\alpha}+d_{\|} \tau_{\alpha}\right)=\tilde{\omega}+d_{\|} \tau
$$

where $\tilde{\omega}=\Sigma_{\alpha} f_{\alpha} \omega_{\alpha} \in \Gamma\left(A_{b}^{* s}\right) \otimes \Lambda^{t}\left(\omega_{1}, \ldots, \omega_{r}\right)_{n}$ and $\tau=\Sigma_{\alpha} f_{\alpha} \tau_{\alpha}$. This shows that $E_{1}(\phi)$ is onto, concluding the proof of the theorem.

As a corollary to the Leray-Hirsch theorem we have the following representation of the map $i_{*}$.

COROLlARY. Let $\mathcal{F}$ be a Hausdorff holomorphic, cohomology product foliation with cohomology basis $\omega_{1}, \ldots, \omega_{r}$. Then there is a commutative diagram

$$
\begin{array}{ccc}
H^{1}\left(M, \theta_{\mathcal{F}}\right) & \stackrel{i_{*}}{\rightarrow} & H^{1}\left(M, \mathbf{Q}_{\mathcal{F}}\right) \\
\hdashline \uparrow & & \ddots \uparrow \\
H^{1}\left(A_{b}^{*}, d_{b}\right) \oplus \Gamma\left(\theta_{\mathcal{F}}\right) \otimes \Lambda^{1}\left(\omega_{1}, \ldots, \omega_{r}\right)_{n} & \stackrel{j^{*}}{\rightarrow} & \Gamma\left(\mathbf{Q}_{\mathcal{F}}\right) \otimes \Lambda^{1}\left(\omega_{1}, \ldots, \omega_{r}\right)_{n}
\end{array}
$$

Hence, $\mathscr{F}$ is infinitesimally stable under holomorphic deformations if and only if $\Gamma\left(\theta_{\mathscr{G}}\right)=0$.

It is natural to ask if there is a naturally occurring class of foliations for which $\Gamma\left(\theta_{\mathcal{G}}\right)=0$. In [2] we showed that the construction of the Kobayashi metric on complex manifolds can be extended to yield a metric on the leaf space of a holomorphic foliation. If this metric is nondegenerate the foliation is called hyperbolic. The well-known result that compact hyperbolic manifolds have no holomorphic vector fields [9] generalizes as follows.

LEMMA. If $\mathcal{F}$ is a hyperbolic foliation on a compact $C^{\infty}$-manifold $M$ and if there is $a C^{\infty}$-foliation transverse to $\mathcal{F}$ then $\Gamma\left(M, \theta_{\mathcal{G}}\right)=0$.

Proof. Suppose $X \in \Gamma\left(M, \theta_{\mathcal{G}}\right)$ is nonzero and let $N \hookrightarrow M$ be a leaf of the foliation transverse to $\mathscr{F}$. Then $N$ is a hyperbolic manifold (the complex structure on $N$ is induced by that of $\mathscr{F}$ and that $N$ is hyperbolic follows from the definition of hyperbolic foliation [2]). The foliation transverse to $\mathscr{F}$ defines a splitting $T M=L \oplus Q$ so that $Q$ is the distribution of the transverse foliation. Therefore, the identification $Q^{(1,0)} \cong Q$ allows us to identify $X$ with a vector field on $M$ which is tangent to $N$. Since $M$ is compact the vector field $X$ is complete and hence restricts to a complete, holomorphic vector field on $N$. As in the proof of the corresponding theorem for hyperbolic manifolds [9] we can use $X_{\mid N}$ to define an immersion of a complex line in $N$. To do this observe that $[X, \sqrt{-1} X]=0$ so if $\nu_{t}$ is the flow of $X$ and $\mu_{t}$ is the flow of $\sqrt{-1} X$ then $\nu_{t} \circ \mu_{s}=\mu_{s} \circ \nu_{t}$. Let $x_{0} \in N$ be a point at which $X$ is nonzero; then define the immersion by 


$$
\begin{aligned}
\mathbf{C} & \rightarrow N \\
a+\sqrt{-1} & b \mapsto \mu_{b}\left(v_{a}\left(x_{0}\right)\right) .
\end{aligned}
$$

Since the Kobayashi metric of $\mathbf{C}$ is $\mathbf{0}$, by the distance decreasing property of holomorphic maps, the manifold $N$ cannot be hyperbolic. This contradiction proves the theorem.

The following theorem is an immediate consequence of Theorem 1 and the above results.

THEOREM 2. Let $\mathcal{F}$ be a holomorphic foliation on a compact manifold $M$. Suppose that there is an $\mathrm{SL}(p)$-foliation transverse to $\mathcal{F}$ and that $\mathscr{F}$ is a cohomology product foliation. Then if $\mathscr{F}$ is hyperbolic it follows that $\mathcal{F}$ is stable under holomorphic deformations.

We will now apply these results to the case where $M$ is the total space of a $G$-principal fiber bundle over a complex manifold $N$. Specifically, let $M \stackrel{\pi}{\rightarrow} N$ be a $G$-principal bundle and let $\mathcal{F}$ be the foliation of $M$ by the fibers of $\pi$ with complex structure induced by the complex structure on $N$. Let $w T M \rightarrow g$ be a connection form whose curvature lies in $\Gamma\left(Q^{(1,0)^{*}} \wedge T^{*} M_{\mathrm{C}} \otimes \mathrm{g}\right)$. If $M \rightarrow N$ is holomorphic such a choice of connection can always be made. Then the induced map

$$
w^{*}: \Lambda \cdot g^{*} \rightarrow \Gamma\left(\Lambda \cdot T^{*} M_{\mathbf{C}}\right)
$$

takes closed, left invariant forms on $G$ to forms whose differentials lie in $\Gamma\left(Q^{(1,0)^{*}} \wedge \Lambda \cdot T^{*} M_{\mathbf{C}}\right)$ and hence $\mathcal{F}$ is a cohomology product foliation. If the connection $w$ is flat then the horizontal distribution defines an $\operatorname{SL}(p)$-foliation transverse to $\mathscr{F}$. Hence we have the following theorem.

THEOREM 3. Let $\mathcal{F}$ be a holomorphic foliation on $M$ given as above. Then $\mathcal{F}$ is infinitesimally stable under holomorphic deformations if and only if there are no holomorphic vector fields on $N$. Furthermore if $M$ is compact and the connection $w$ is flat then $\mathcal{F}$ is stable under holomorphic deformations if there are no holomorphic vector fields on $N$.

\section{BIBLIOGRAPHY}

1. T. Duchamp and M. Kalka, Deformation theory for holomorphic foliations, J. Differential Geometry (to appear).

2. 117-122.

, Holomorphic foliations and the Kobayashi metric, Proc. Amer. Math. Soc. 67 (1977),

3. R. Edwards, K. Millett and D. Sullivan, On foliations with compact leaves, Topology 16 (1977), 13-32.

4. D. B. A. Epstein, Foliations with all leaves compact, Ann. Inst. Fourier (Grenoble) 26 (1976), 265-282.

5. D. B. A. Epstein and H. Rosenberg, Stability of compact foliations, University of Warwick (preprint).

6. R. S. Hamilton, Deformation theory for foliations (preprint).

7. J. Heitsch, A cohomology for foliated manifolds, Comment Math. Helv. 50 (1975), 197-218.

8. F. Kamber and P. Tondeur, Invariant differential operators and the cohomology of Lie algebra sheaves, Mem. Amer. Math. Soc. No. 113, 1971. 
9. S. Kobayashi, Hyperbolic manifolds and holomorphic maps, Dekker, New York, 1970.

10. R. Langevin and H. Rosenberg, On stability of compact leaves and fibrations, Topology 16 (1977), 107-112.

11. B. Reinhart, Harmonic integrals on almost product manifolds, Trans. Amer. Math. Soc. 88 (1958), 243-275.

12. __ Harmonic integrals on foliated manifolds, Amer. J. Math. 81 (1959), 529-536.

Department of Mathematics, University of Utah, Salt Lake CtTy, Utah 84112

Department of Mathematics, The Johns Hopknns University, Baltimore, Maryland 21218

Current address (T. Duchamp): Department of Mathematics, University of Washington, Seattle, Washington 98195

Current address (M. Kalka): Department of Mathematics, Tulane University, New Orleans, Louisiana 70118 